\title{
Stability Analysis of Conventional Rocket Model using CFD Tool
}

\author{
Hari Prasanna Manimaran \\ Department of Aeronautical Engineering \\ Kaunas university of technology \\ Kaunas, Lithuania
}

\author{
Gopi Kompelli \\ Department of Vehicle Engineering \\ Kaunas University of technology \\ Kaunas, Lithuania
}

\author{
Khaja Naib Rasool Shaik \\ Department of Aeronautical Engineering \\ Kaunas University of technology \\ Kaunas, Lithuania
}

\begin{abstract}
This research paper investigates the methods of finding the centre of pressure and centre of gravity of conventional rocket body to predict the stability. Determining stability is crucial for the rocket ballistics. As the stable rocket body archives the higher accuracy and obtains the required performance. Centre of gravity can be calculated by experimental methods and the centre of pressure can be calculated using the cross-sectional area. But using computational method is more convenient. In this research computational software are used to determine the stability of the rocket model.
\end{abstract}

Keywords - Rocket, stability analysis, rocket ballistics, computational fluid dynamics.

\section{INTRODUCTION}

Rocket is the tubular body, which changes its attitude, or wobbles due to the cross gust of wind hits the body of the rocket or due to thrust instabilities. All object rotates about its centre of gravity CG [1]. During the rotation, the gust hits the body at some angle $\boldsymbol{\alpha}$ to the central axis. Lift is generated due to the inclination angle by the fins and body [2]. While the drag remains constant. Both lift and drag act on the CG [3]. Any rocket, which is about to fire should to stable during its flight.

The rocket will return to its initial condition if it is disturbed when the centre of gravity CG is kept above the centre of pressure CP. CP is the location where all aerodynamics forces act. This restoring force causes the rocket to be stable.

Stability can be obtained by increasing the distance between the CG and CP. Minimum of one calibre is required, which is the distance of diameter of the rocket body [4]. Another way is increasing the surface area exposed to the air by introducing fins. Therefore, the analysis of stability is crucial for rocket design [5]. Computational methods are more convenient in obtaining the location of $\mathrm{CG}$ and $\mathrm{CP}$. This research aims to find the location of the CG and CP of the cad-modelled rocket in order to optimize for stability [6].

\section{ROCKET MODELLING}

\section{A. Modelling in SolidWorks}

Rocket body is modelled in SolidWorks software as a thinwalled body with a thickness of $2 \mathrm{~mm}$. The total length is $1311 \mathrm{~mm}$ with the outer average diameter of $140 \mathrm{~mm}$. The material is selected as Aluminum. For trajectory control, the rocket uses different types of fins. Here, for this rocket, a traditional Clipped delta fin has been modelled. The mass is given at the nose cone to increase the centre of gravity towards the nose. The nose is shaped similarly as elliptical and hemispherical with the enlarged body at the nose for payload. The mass of the entire rocket is predetermined. As the first stage of the rocket has multiple boosters, these boosters are simplified as the single large cylindrical shape for the convenience and reduce the iteration time. The four fins are arranged in equal interval of the angles.

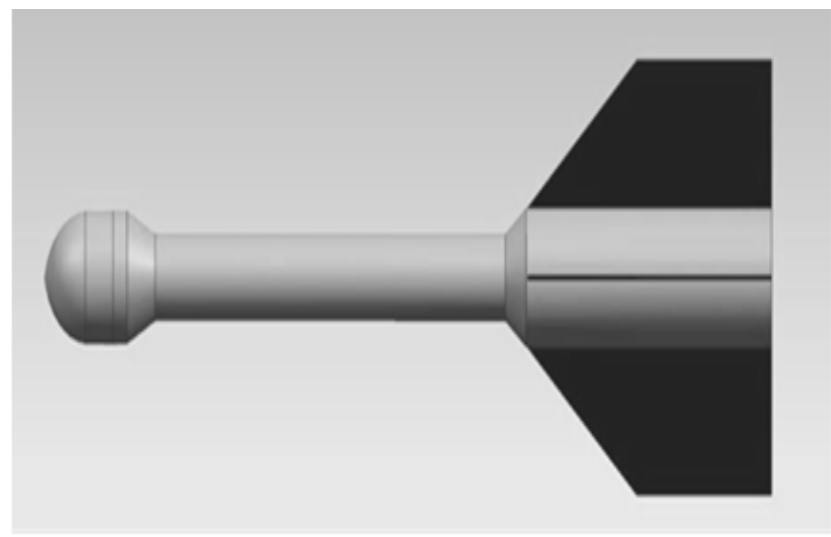

Fig. 1, computer aided design model of rocket

Table 1. Properties of the model

\begin{tabular}{|c|c|}
\hline Volume & $3809228.697931916 \mathrm{~mm} 3$ \\
\hline Area & $1275609.362754997 \mathrm{~mm} 2$ \\
\hline Mass & $29.828698611 \mathrm{~kg}$ \\
\hline Density & $0.000007831 \mathrm{~kg} / \mathrm{mm} 3$ \\
\hline Weight & $292.519607235 \mathrm{~N}$ \\
\hline Radius of gyration & $476.786300386 \mathrm{~mm}$ \\
\hline Centre of mass & $470.808811965 \mathrm{~mm}$ \\
\hline
\end{tabular}

\section{DETERMINING THE CENTRE O PRESSURE}

The modelled rocket is analyzed in the fluid flow, it requires a fluid domain. Figure .2 shows the fluid domain modelled around the rocket with merging the two solids. The two bodies, 
Rocket and the Fluid domain extruded is subtracted from each other to obtain two distinctive bodies using the Subtract subcommand. This forms the shape of a cavity in the solid body.

Subtract command is used to cut halfway through the overall model design which can be analyzed for flow characteristics and other parameters using ANSYS FLUENT software.

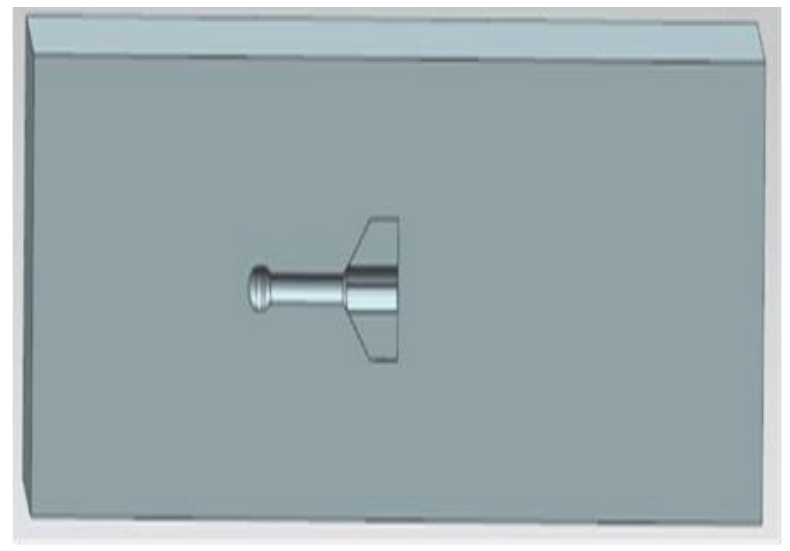

Fig. 2, Rocket model inside the domain

The solid design is saved in IGS format to be analyzed in fluent.

The solid-works file is imported in the fluent, geometry workbench and the rocket body and the fluid domain is named as "Rocket" and "fluid environment". Then the two solids are divided using subtract command from Create-Boolean-from operation select subtract. The rocket body is selected as a tool body and the fluid environment as the target body. the Ansys fluent environment is shown in figure. 3 .

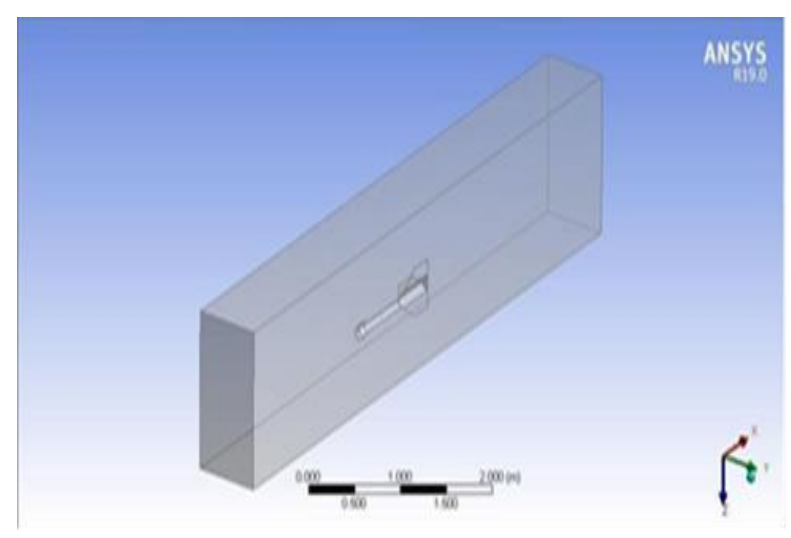

Fig. 3, Model imported in Ansys modeler

Then the model is loaded in Mesh environment. Initially, the part is to be named. The front wall of the fluid domain is named as "INLET" the counter side wall is named as "OUTLET", the four-side wall is named as "surroundings". Then the model has meshed for accurate fluid analysis. The relevance centre is given "fine", so the density of mesh is higher near the wall or the surface of the body. Tetrahedron type mesh is applied.

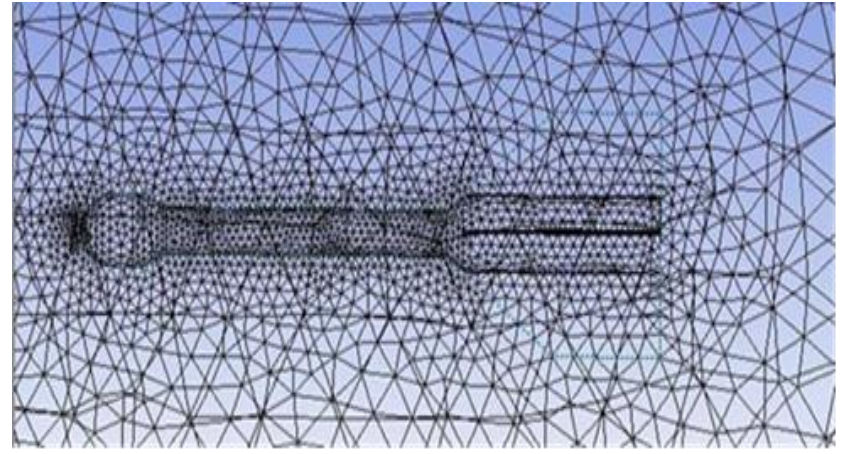

Fig. 4, Meshed model

\section{CFD ANALYSIS}

\section{A. Preprocessing}

Preprocessing: Once the mesh is generated and the project is saved, the next procedure is to define the models, material and the boundary condition values

1) Model: viscous model-Transition (SST)

2) Material: Fluid-Air (the environment is considered as air)

\section{3) Boundary condition}

a) Inlet: velocity- $800 \mathrm{~m} / \mathrm{s}$ (the flow of air is at the given velocity and the rocket is considered static)

b) Outlet: Pressure

c) Symmetry, rocket and wall settings remain default.

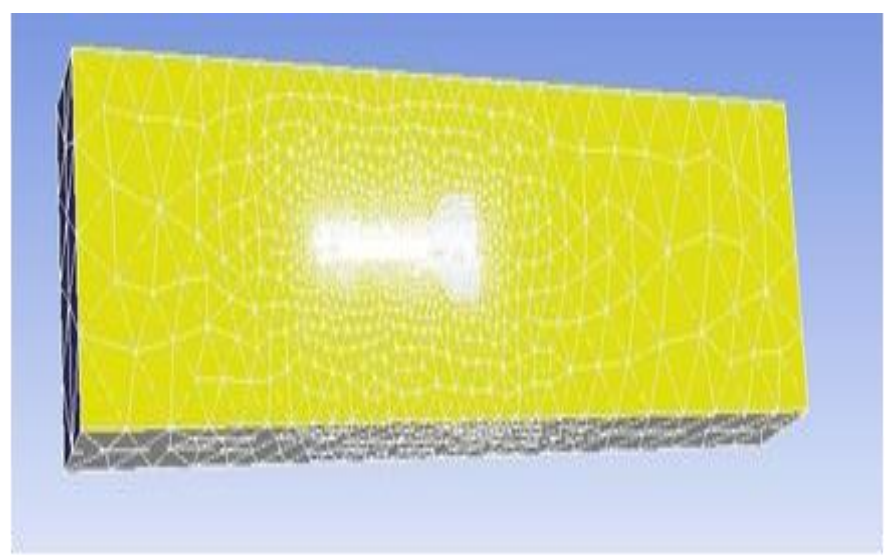

Fig. 5, Model in pre-process

Once the setup settings are done. The next procedure is to perform the Initialization and running the calculations. This is done in the four steps of the Analysis Process under Solution.

\section{B. Solution}

1) Initialization: Hybrid Initialization

2) Run Calculation:

a) Number of Iterations: 100

b) Reporting Interval: 10 


\section{Post-processing}

The iteration graph shows the results graph with converging values.

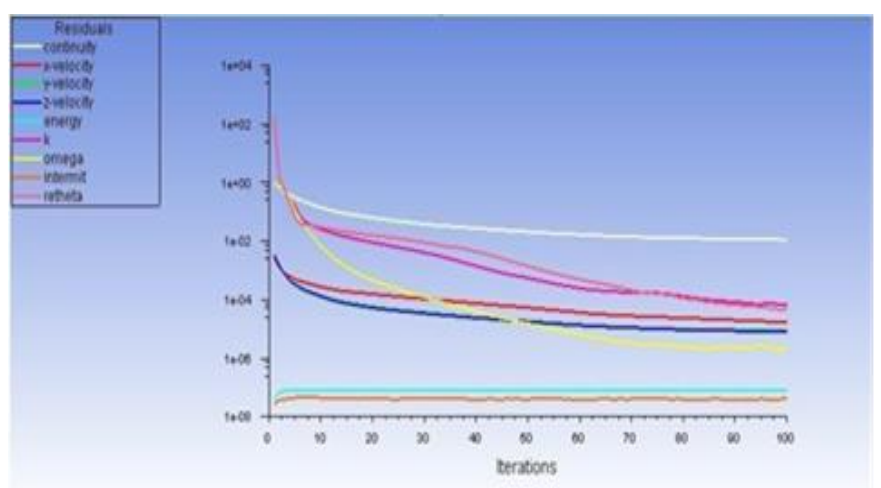

Fig. 6, Iteration graph

The post-processing image highest pressure coefficient exists at the nose and the leading edge of the fin. These are well displayed in figure.7. the colour gradient shows the highest pressure with the red and lowest pressure with blue.

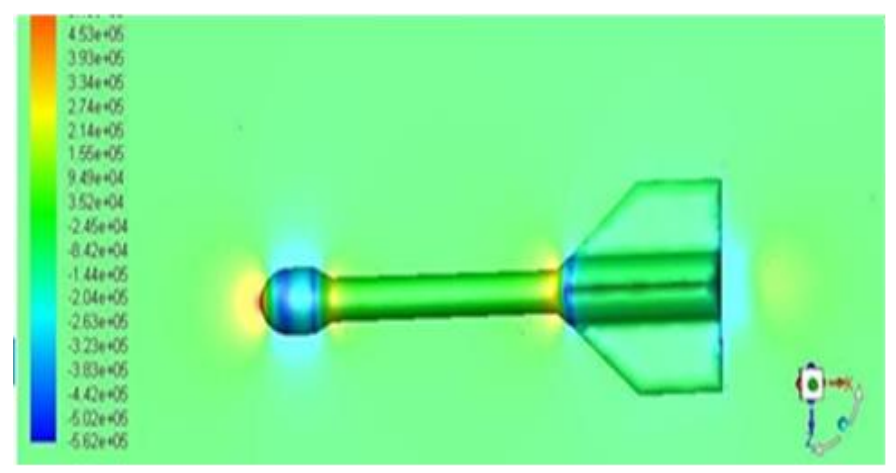

Fig. 7, Determining pressure coefficient

figure. 8 shows the velocity magnitude of the model the colour code shows the red for the highest velocity of the fluid and the blue remains the lowest. The velocity around the rocket is higher and especially the sides of the nose cone have the highest velocity.

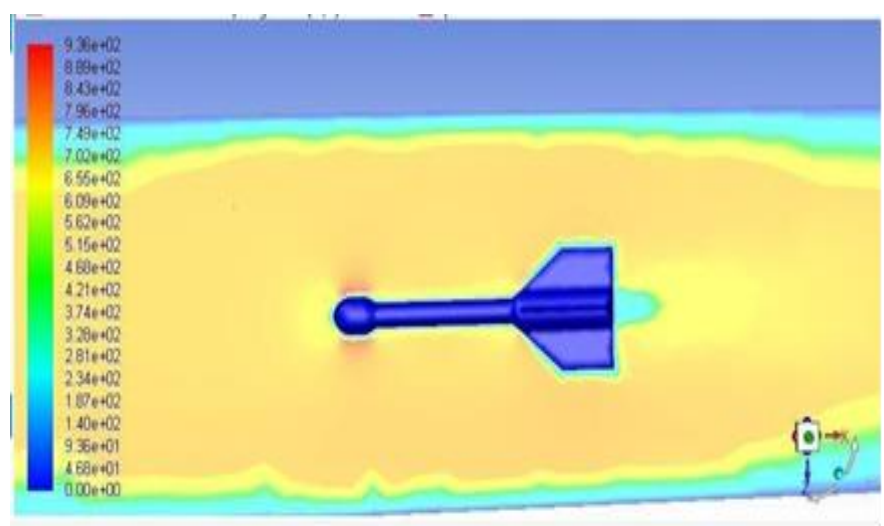

Fig. 8, determining velocity magnitude

Figure. 9 shows the flow of velocity vector around the model rocket. This gives a clear picture of the velocity vector motion and gives detail visualization.

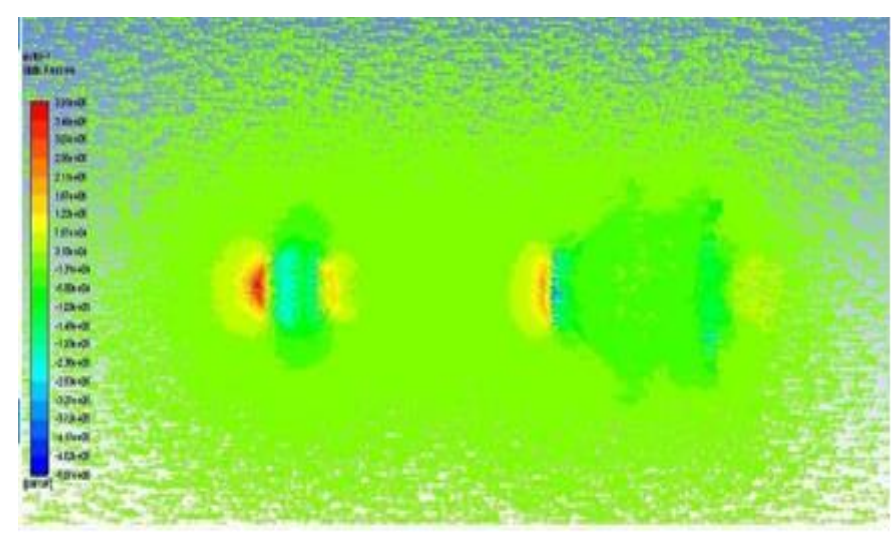

Fig. 9, Velocity vector

Figure.10, show the expression tab where the equation to find the centre pressure is to be given.

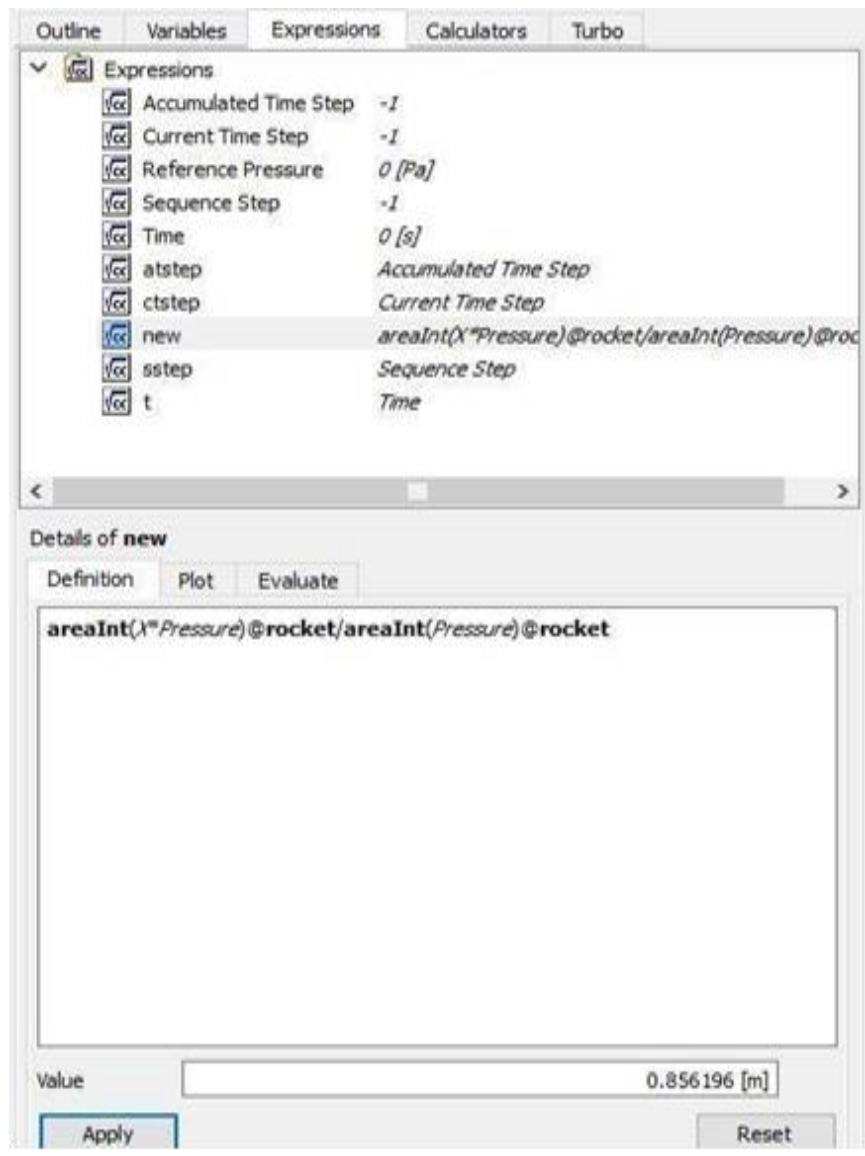

Fig. 10, Expression tab with stability equation

Once the calculation is completed, the Centre of the pressure of the rocket in normal and with vicious force is found by using the given expressions under the solution window.

To input expressions, open results segment: Right-click on expressions tab, new expressions and name it. In the below definition box type the expression and obtain the result. 


\section{RESULTS}

The Coefficient of pressure is obtained for the various expressions:

arealnt( $X^{*}$ Pressure)@rocket/arealnt(Pressure)@rocket

$\mathbf{C P X}=\mathbf{8 5 6} \mathbf{~ m m}$

arealnt(Y*Pressure)@rocket/arealnt(Pressure)@ rocket

$\mathbf{C P Y}=\mathbf{- 5 9} \mathbf{~ m m}$

arealnt(Z*Pressure)@rocket/arealnt(Pressure)@rocket

$\mathrm{CPZ}=\mathbf{1 - 4} \mathrm{mm}$

The Following results were obtained with the viscous forces expressions:

$\operatorname{arealnt}\left(X^{*}(\right.$ pressure + wall

shear))@rocket/arealnt((pressure+wallshear))@ rocket

viscous force $X=856 \mathrm{~mm}$

arealnt $\left(Y^{*}\right.$ (pressure+wall

shear))@rocket/arealnt((pressure+wallshear))@ rocket

viscous force $Y=59 \mathrm{~mm}$

arealnt $\left(Z^{*}\right.$ (pressure + wall

shear))@rocket/arealnt((pressure+wall shear ))@rocket

\section{viscous force $Z=1.4 \mathrm{~mm}$}

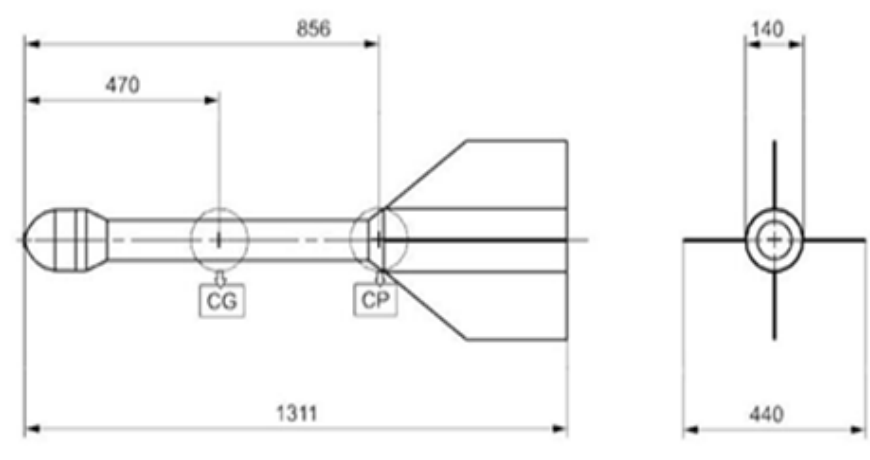

Fig. 11, Location of Centre of pressure and centre of gravity

The centre of Gravity is located at $470 \mathrm{~mm}$ from the nose tip. The centre of Pressure is located at $856 \mathrm{~mm}$. Centre of pressure is located at $386 \mathrm{~mm}$ behind the centre of gravity. The diameter of the airframe is $140 \mathrm{~mm}$. the location of CG and CP shows that this rocket is $\mathbf{2 . 7 5}$-body calibre stable. The stability of the projectile is measured in terms of calibre. The calibre is the value obtained by dividing the distance between the centre of pressure and centre of gravity and divided by the diameter of the projectile.

\section{CONCLUSION}

the distance between the centre pressure and centre of gravity plays a vital role in the stability of the rocket. During the motion of the rocket, it uses the propellant to burn to produce thrust. As the mass of the propellant being ejected as a combustion product, the centre of gravity moves from the initial location. But the centre of pressure remains constant as there is no reduction in cross-sectional area. This makes the distance between CG and CP changes and alters the stability.
Thus the stability should be kept higher calibre to make sure the change in CG will not affect the stability of the rocket in the trajectory. The following results are obtained on this research is Centre of gravity: $470 \mathrm{~mm}$; Centre of pressure: $856 \mathrm{~mm}$; from this Analysis, the CG and CP are calculated, and it is observed that the rocket is stable with 2.75 calibre stability.

\section{REFERENCE}

[1]. Tom Benson, "Rocket stability condition," NASA, 12 June 2014 [Online]. Available: https://www.grc.nasa.gov/www/k12/rocket/rktstabc.html. [Accessed 2019].

[2]. C. Herschel, "Aerothermodynamic design problems of the hypersonic flight vehicle," American institute of aeronautics and astronautics, 2009.

[3]. R.\&J.B\&N.B\&C.L\&H.F.Li, "Drag reduction mechanisms of a Car model at moderate yaw by bi-frequency forcing," Physical review fluids, 2019.

[4]. L. a. C.Lin, "Investigation of the stability of the laminar boundary layer," NASA Tech Docs, NASA, 1946.

[5]. T.S.Taylor, Introduction to rocket science and engineering, London: CRC Press, 2009.

[6]. E. V. Eijkhout, Introduction to high-performance scientific computing, Texas, 2011.

[7]. Buckland, M., \& Gey, F. The relationship between recall and precision. Journal of the American Society for Information Science, 45, 12-19, 1994.

[8]. Borgman, C.L. (Ed.). Scholarly Communication and Bibliometrics. London: Sage, 1990.

[9]. Bauin, S., \& Rothman, H. (1992). "Impact" of journals as proxies for citation counts. In P. Weingart, R. Sehringer \& M. Winterhager (Eds.), Representations of Science and Technology (pp. 225-239). Leiden: DSWO Press, 1992.

[10]. Hoppe, K., Ammersbach, K., Lutes-Schaab, B., \& Zinsmeister, G. Express: An experimental interface for factual information retrieval. In J.-L. Vidick (Ed.), Proceedings of the 13th International Conference on Research and Development in Information Retrieval (ACM SIGIR '91) (pp. 63-81). Brussels: ACM. 1990

[11]. Kling, R., \& Elliott, M. Digital library design for usability. Retrieved December 7, 2001, from http://www.csdl.tamu.edu/DL94/paper/kling.html. 1994. 